\title{
Crystallization of magnetic dipolar monolayers: a density functional approach
}

\author{
Sven van Teeffelen $\ddagger$ Hartmut Löwen, and Christos N. Likos \\ Institut für Theoretische Physik II: Weiche Materie, \\ Heinrich-Heine-Universität Düsseldorf, Universitätsstraße 1, D-40225 Düsseldorf, \\ Germany
}

\begin{abstract}
We employ density functional theory to study in detail the crystallization of super-paramagnetic particles in two dimensions under the influence of an external magnetic field that lies perpendicular to the confining plane. The field induces nonfluctuating magnetic dipoles on the particles, resulting into an interparticle interaction that scales as the inverse cube of the distance separating them. In line with previous findings for long-range interactions in three spatial dimensions, we find that explicit inclusion of liquid-state structural information on the triplet correlations is crucial to yield theoretical predictions that agree quantitatively with experiment. A nonperturbative treatment is superior to the oft-employed functional Taylor expansions, truncated at second or third order. We go beyond the usual Gaussian parametrization of the density site-orbitals by performing free minimizations with respect to both the shape and the normalization of the profiles, allowing for finite defect concentrations.
\end{abstract}

PACS numbers: $64.10 .+\mathrm{h}, 64.70 . \mathrm{Dv}, 82.70 . \mathrm{Dd}$

$\ddagger$ Corresponding author. E-mail: teeffelen@thphy.uni-duesseldorf.de 


\section{Introduction}

Classical density functional theory (DFT) is the method of choice to the study of inhomogeneous fluids [1]. Perhaps the most extreme inhomogeneities arise in a crystalline solid, where the density field $\rho(\boldsymbol{r})$ is both periodic and shows extreme differences between its local values on the lattice sites and in the interstitial regions. DFT has been successfully applied to the problem of crystallization of a number of different systems [2, 3, 4, 5, 6], mostly in three spatial dimensions. Here, the most popular system is the prototype of hard spheres, for which a geometry-based theory [7, 8, 9] has proven quite successful. For soft interactions [10, 11, 12], however, where one cannot assign geometrical measures to the interacting point-particles, one has to resort to other functionals. In particular, it has been shown [13] that for long-range interactions, structural information of the liquid on the pair-level is insufficient and triplet fluid correlations should be allowed to explicitly flow into the construction of the functional. Even less is known for crystallization in two spatial dimensions [14, 15, 16, 17]. Here, we consider a combination of the two above-mentioned cases in considering long range interactions in two spatial dimensions and we study in detail the role played by accurate liquid-state information on triplet correlations in determining phase boundaries between a fluid and the coexisting crystal.

In this paper, we study freezing of a classical two-dimensional model fluid, namely of a fluid of aligned dipoles directed perpendicular to the 2D-plane and repelling each other with a soft $1 / r^{3}$ inverse-power pair potential, with the help of density functional theory (DFT). In Ref. [17] we studied freezing of the the dipolar system with the modified weighted density approximation and its extension to third order correlation functions. Within this paper we will extend our previous study in several ways: We allow for a finite defect concentration and relax the constraint of Gaussian density peaks in the crystalline phase, as, e.g., suggested for hard sphere crystals in Ref. [18]. Furthermore, we systematically study the influence of perturbative and non-perturbative inclusion of higher order correlation functions of the liquid in the density functional approximation on the freezing transition. We employ two different approximations to the three-particle correlation functions, which lead to substantially different results, therefore signalling the importance of an accurate approximation of the latter.

We use different approximations to the DFT - based on the famous and powerful approach by Ramakrishnan and Yussouff [19], but extending on the latter in taking higher-order terms into account, as will be described below. The quantity to be approximated in the DFT of freezing is the excess Helmholtz free energy functional $F_{\text {ex }}[\rho(\boldsymbol{r})]$, a unique functional of the inhomogeneous one-particle density $\rho(\boldsymbol{r})$ of the solid [1]. The uniqueness property implies that the excess free energy can be formally expanded about the excess free energy of a homogeneous fluid at a uniform density $\rho$ in terms of density difference $\Delta \rho(\boldsymbol{r})=\rho(\boldsymbol{r})-\rho$ :

$\beta F_{\mathrm{ex}}[\rho(\boldsymbol{r})]=\beta F_{\mathrm{ex}}(\rho)-\sum_{n=1}^{\infty} \frac{1}{n !} \int_{V} \mathrm{~d} \boldsymbol{r}_{1} \ldots \mathrm{d} \boldsymbol{r}_{n} c_{0}^{(n)}\left(\boldsymbol{r}, \ldots, \boldsymbol{r}_{n} ; \rho\right) \Delta \rho\left(\boldsymbol{r}_{1}\right) \ldots \Delta \rho\left(\boldsymbol{r}_{n}\right)$, 
where $\beta=1 /\left(k_{B} T\right)$ and $V$ is the volume occupied of the system. $F_{\text {ex }}(\rho)$ is the Helmholtz excess free energy and the $c_{0}^{(n)}$ are the $n$-particle direct correlation functions of the fluid, which are well known up to second order for dipolar fluids [17].

Within the theory of Ramakrishnan and Yussouff this series expansion is truncated at second order. We therefore refer to the theory as "second order theory" (SOT). Part of the reason for this truncation lies in poor knowledge about higher than second order correlation functions; the truncation is not well justified in the problem of freezing, since here $\Delta \rho$ is not a small parameter. In particular, it has been extensively shown that the SOT fails to accurately predict freezing for systems interacting via long-range pair potentials for three-dimensional systems [13, 20]. We will show in this work, that also for the two-dimensional dipolar system the SOT highly underestimates the stability of the crystal. Therefore, several approaches have been employed to include higher than second-order terms in the expansion - in a perturbative [10] or non-perturbative way [11, 13, 21, 22].

The simplest attempt to go beyond the SOT is to explicitly include the third order term in the expansion in equation (1), which we refer to as "third order theory" (TOT). Employing the TOT demands an approximate form of the three-particle direct correlation function $c_{0}^{(3)}\left(\boldsymbol{r}, \boldsymbol{r}^{\prime} ; \rho\right)$ of the fluid. We will show here, that - given an accurate expression for $c_{0}^{(3)}\left(\boldsymbol{r}, \boldsymbol{r}^{\prime} ; \rho\right)$-including this term substantially improves the predicted freezing temperature of the long-range $1 / r^{3}$-fluid (in line with previous findings for long-range interactions in 3D [23]).

A third approach to the DFT we follow here, is the Modified Weighted-Density Approximation (MWDA) [21] by Denton and Ashcroft which we already presented for the dipolar system in brief in a previous paper [17]. This approach includes first and second order correlation functions of the fluid exactly (as in the SOT) and higher order correlation functions in a non-perturbative, implicit fashion. We find that the MWDA, in two dimensions, slightly shifts the freezing transition to higher temperature as compared to the SOT, still highly underestimating the stability of the solid state. In a fourth approach we employ the so called "extended modified weighted-density approximation" (EMA), as suggested in Refs. [13, 22]. Different from the MWDA, this approximation to the density functional now includes not only first and second, but also third order correlation functions of the fluid exactly (as in the TOT). Higher than third-order correlation functions are contained in a nonperturbative, implicit fashion, following a similar scheme as in the MWDA. For the dipolar system we find that this approach leads to a very accurate value of the freezing transition temperature, lying slightly above the one obtained from the simpler TOT. The two-particle correlation functions of the liquid are obtained from liquid state integral equation theory and from simulation. The three-particle correlation functions are obtained applying two approximations, both based on the two-particle correlation functions: The first approximation used is by Denton and Ashcroft (DA) [24], and the second is by Barrat, Hansen, and Pastore (BHP) [25].

We find that the inclusion of higher order correlation functions in a perturbative 
(TOT) or non-perturbative (EMA) way subsequently increase the freezing transition temperature, thus broadening the range of the thermodynamical stability of the crystal. In fact, we find the freezing transition temperature to be in good agreement with experiment [26] and simulation [27, 28, 29]. The importance of inclusion of third order correlation functions is addressed to the long-range nature of the dipole-dipole pair interaction.

The rest of this work is organized as follows: In section 2 we give a brief description of the MWDA and of the EMA. In section 3 we apply the different approximations to the DFT to freezing of monodispers two-dimensional liquids. The theory is adapted to the dipolar system under study in section 4 . In section 5 we present the resulting phase diagrams and different structural properties of the crystalline system, and we conclude in section 6.

\section{Modified weighted-density approximation and its extension to third-order correlation functions}

It is well known that the intrinsic Helmholtz free energy of an inhomogeneous system can be divided into an "ideal" and an "excess" part,

$$
F[\rho(\boldsymbol{r})]=F_{\text {id }}[\rho(\boldsymbol{r})]+F_{\mathrm{ex}}[\rho(\boldsymbol{r})] .
$$

The "ideal" term

$$
F_{\text {id }}[\rho(\boldsymbol{r})]=\beta^{-1} \int \mathrm{d} \boldsymbol{r} \rho(\boldsymbol{r})\left\{\ln \left[\rho(\boldsymbol{r}) \Lambda^{2}\right]-1\right\},
$$

is known exactly. In equation (3) $\Lambda$ is the thermal de Broglie wavelength. The excess part can only be calculated approximately. In contrast to the SOT and TOT, within the MWDA and EMA the excess free energy of the inhomogeneous system is approximated by setting it equal to the excess free energy of a uniform liquid evaluated at a weighted density $\hat{\rho}$,

$$
F_{\mathrm{ex}}[\rho(\boldsymbol{r})] \approx F_{\mathrm{ex}}^{\mathrm{M} / \mathrm{E}}[\rho(\boldsymbol{r})]=N f_{0}\left(\hat{\rho}^{\mathrm{M} / \mathrm{E}}\right),
$$

where superscripts denote the approximations to the DFT, MWDA (M) and EMA (E), respectively. $N$ is the number of particles in the system and $f_{0}(\hat{\rho})$ is the excess free energy per particle of the liquid at the weighted density $\hat{\rho}$. The latter is expressed as

$$
\begin{aligned}
\hat{\rho}^{\mathrm{M} / \mathrm{E}}[\rho(\boldsymbol{r})]= & \frac{1}{N} \int \mathrm{d} \boldsymbol{r} \mathrm{d} \boldsymbol{r}^{\prime} \rho(\boldsymbol{r}) \rho\left(\boldsymbol{r}^{\prime}\right) w\left(\boldsymbol{r}-\boldsymbol{r}^{\prime} ; \hat{\rho}\right) \\
& +\frac{1}{N^{2}} \int \mathrm{d} \boldsymbol{r} \mathrm{d} \boldsymbol{r}^{\prime} \mathrm{d} \boldsymbol{r}^{\prime \prime} \rho(\boldsymbol{r}) \rho\left(\boldsymbol{r}^{\prime}\right) \rho\left(\boldsymbol{r}^{\prime \prime}\right) v\left(\boldsymbol{r}-\boldsymbol{r}^{\prime}, \boldsymbol{r}-\boldsymbol{r}^{\prime \prime} ; \hat{\rho}\right),
\end{aligned}
$$

where the second term only appears in the EMA and not in the MWDA. The weight functions $w(\boldsymbol{r} ; \rho)$ and $v\left(\boldsymbol{r}, \boldsymbol{r}^{\prime} ; \rho\right)$ are determined in such a way that the approximate functional $F_{\mathrm{ex}}^{\mathrm{M} / \mathrm{E}}[\rho(\boldsymbol{r})]$ is exact up to second (MWDA) or third (EMA) order in density difference $\Delta \rho(\boldsymbol{r})=(\rho(\boldsymbol{r})-\rho)$, i.e., up to that order equation (4) and equation (1) do agree. Note that the weighted density $\hat{\rho}$ is determined self-consistently, as it appears as 
an argument of both weight functions. In order to obtain equality with equation (1) up to second or third order in $\Delta \rho$ we demand the weight functions to be normalized, i.e.,

$$
\int_{V} \mathrm{~d} \boldsymbol{r} w(\boldsymbol{r} ; \rho)+\frac{1}{V} \int_{V} \mathrm{~d} \boldsymbol{r} \int_{V} \mathrm{~d} \boldsymbol{r}^{\prime} v\left(\boldsymbol{r}, \boldsymbol{r}^{\prime} ; \rho\right)=1 .
$$

and to fulfill the requirements

$$
\begin{aligned}
& \lim _{\rho(\boldsymbol{r}) \rightarrow \rho}\left[\frac{\delta^{2} F_{\mathrm{ex}}^{\mathrm{M} / \mathrm{E}}}{\delta \rho(\boldsymbol{r}) \delta \rho\left(\boldsymbol{r}^{\prime}\right)}\right]=-\beta^{-1} c_{0}^{(2)}\left(\boldsymbol{r}-\boldsymbol{r}^{\prime} ; \rho\right), \\
& \lim _{\rho(\boldsymbol{r}) \rightarrow \rho}\left[\frac{\delta^{3} F_{\mathrm{ex}}^{\mathrm{E}}}{\delta \rho(\boldsymbol{r}) \delta \rho\left(\boldsymbol{r}^{\prime}\right) \delta \rho\left(\boldsymbol{r}^{\prime \prime}\right)}\right]=-\beta^{-1} c_{0}^{(3)}\left(\boldsymbol{r}-\boldsymbol{r}^{\prime}, \boldsymbol{r}-\boldsymbol{r}^{\prime \prime} ; \rho\right),
\end{aligned}
$$

where $c_{0}^{(2)}(\boldsymbol{r} ; \rho)$ and $c_{0}^{(3)}\left(\boldsymbol{r}, \boldsymbol{r}^{\prime} ; \rho\right)$ are the two- and three-particle correlation functions of the liquid with density $\rho$ which are an input to the theory. These conditions uniquely determine the weight functions. In order to obtain the simple algebraic equations for $v$ and $w$ that can be found in Ref. [22] a further approximation has to be made: The inner integral in the second term of equation (6) is assumed to be equal to a constant, $C$ (demanding the first term in equation (6) to be equal to $1-C$ ), where $C$ is independent of the fixed space coordinate of the weight function $v\left(\boldsymbol{r}, \boldsymbol{r}^{\prime} ; \rho\right)$. The weighted density $\hat{\rho}$ in equation (5) is independent of the choice of $C$ [22].

For non-zero wave vectors $\left(\boldsymbol{k} \neq 0, \boldsymbol{k}^{\prime} \neq 0\right.$, or $\left.\boldsymbol{k}+\boldsymbol{k}^{\prime} \neq 0\right)$, the Fourier transforms of the weight functions $\tilde{w}(k ; \rho)$ and $\tilde{v}\left(\boldsymbol{k}, \boldsymbol{k}^{\prime} ; \rho\right)$ are simply proportional to the Fourier transforms of the second- and third-order direct correlation functions $\tilde{c}_{0}^{(2)}(k ; \rho)$ and $\tilde{c}_{0}^{(3)}\left(\boldsymbol{k}, \boldsymbol{k}^{\prime} ; \rho\right)$, respectively:

$$
\begin{aligned}
& -\beta^{-1} \tilde{c}_{0}^{(2)}(k ; \rho)=2 f_{0}^{\prime}(\rho) \tilde{w}(k ; \rho), \\
& -\beta^{-1} \tilde{c}_{0}^{(3)}\left(\boldsymbol{k}, \boldsymbol{k}^{\prime} ; \rho\right)=6 f_{0}^{\prime}(\rho) \tilde{v}\left(\boldsymbol{k}, \boldsymbol{k}^{\prime} ; \rho\right) .
\end{aligned}
$$

Furthermore, equation (5), together with equations (6) and (7) guarantee fulfillment of the sum rules

$$
\begin{aligned}
& \beta^{-1} \tilde{c}_{0}^{(2) \mathrm{M} / \mathrm{E}}(k=0 ; \rho)=2 f_{0}^{\prime}(\rho)+\rho f_{0}^{\prime \prime}(\rho), \\
& \tilde{c}_{0}^{(3) \mathrm{M} / \mathrm{E}}\left(\boldsymbol{k}, \boldsymbol{k}^{\prime}=0 ; \rho\right)=\tilde{c}_{0}^{(3) \mathrm{M} / \mathrm{E}}(\boldsymbol{k},-\boldsymbol{k} ; \rho)=\frac{\partial \tilde{c}_{0}^{(2)}(k ; \rho)}{\partial \rho},
\end{aligned}
$$

where the former is the compressibility sum rule, and where the superscripts on the correlation functions indicate that these functions are the Fourier transforms of the functional derivatives of the approximate excess free energy functionals in the limit of constant average density $\rho$ [c.f. equation (7)]. The primes on the excess free energy density $f_{0}$ denote derivatives with respect to density.

Due to the self-consistency requirement, the approximate excess free energies of both the MWDA and the EMA include contributions from arbitrarily many higher orders. However, if expanded about the excess free energy of a fluid with the same average density as the inhomogeneous system according to equation (1), the MWDA only gives even order terms and estimates the odd order terms zero. Contrary, the EMA includes, approximately, contributions from all higher order terms. In particular, it includes the exact third-order term, which is an input to the theory. 


\section{Application of the different approximations to the DFT to freezing of monodisperse two-dimensional liquids}

In order to find the equilibrium one-particle density $\rho_{\text {eq }}(\boldsymbol{r})$ of a system at a given average density $\rho$ and temperature $T$ we minimize the approximate total free energy functional $F[\rho(\boldsymbol{r})]$ of equation (2) with respect to the inhomogeneous one-particle density $\rho(\boldsymbol{r})$ for fixed $\rho$. As described, for example, in Refs. [21, 22] this minimization is pursued in a number of subsequent steps, depending on the kind of approximation: For all approximations to the DFT, first, an appropriate parametrization for the inhomogeneous one-particle density is made (we will employ a free minimization in section 5.3). Within the SOT and TOT, we can now, in a second step, calculate the excess and ideal parts of the Helmholtz free energy according to equations (1) and (3). However, within the MWDA and EMA, the excess part is given by equation (4), with the weighted density $\hat{\rho}$ obtained in an intermediate step according to equation (5). In a final step, minimization is carried out with respect to all free variables in the parametrization of $\rho(\boldsymbol{r})$.

The crystalline one-particle density which we expect to be in equilibrium for low temperature and/or high density has the symmetry of the triangular crystalthe quadratic lattice is thermodynamically unstable for the whole range of accessible densities/coupling constants and we expect mechanical instability with respect to the triangular lattice for any coupling. We can therefore express $\rho(\boldsymbol{r})$ as a sum over reciprocal lattice vectors (RLV's) of the triangular lattice:

$$
\rho(\boldsymbol{r})=\rho\left[1+\sum_{\boldsymbol{K} \neq 0} \mu_{\boldsymbol{K}} e^{i \boldsymbol{K} r}\right]
$$

where $\rho$ is the average density of the solid, $\{\boldsymbol{K}\}$ is the set of reciprocal lattice vectors (RLV's), and where the $\mu_{\boldsymbol{K}}$ are the dimensionless Fourier components. In terms of Fourier components the excess part to the Helmholtz free energy within SOT and TOT now reads

$$
\begin{aligned}
\beta F_{\mathrm{ex}}^{\mathrm{S} / \mathrm{T}}[\rho(\boldsymbol{r})] / N & =\beta f_{0}(\rho)-\frac{\rho}{2} \sum_{\boldsymbol{K} \neq 0} \mu_{\boldsymbol{K}^{2}}^{2} \tilde{c}_{0}^{(2)}(k ; \rho) \\
& -\frac{\rho^{2}}{6} \sum_{\boldsymbol{K} \neq 0} \sum_{\boldsymbol{K}^{\prime} \neq 0,-\boldsymbol{K}} \mu_{\boldsymbol{K}} \mu_{\boldsymbol{K}^{\prime}} \mu_{-\left(\boldsymbol{K}+\boldsymbol{K}^{\prime}\right)} \tilde{c}_{0}^{(3)}\left(\boldsymbol{K}, \boldsymbol{K}^{\prime} ; \rho\right),
\end{aligned}
$$

the superscript referring to the SOT $(\mathrm{S})$ and to the TOT $(\mathrm{T})$, respectively. The third term only appears in the TOT.

Within the MWDA and EMA, the weighted density, equation (5), now reads

$\hat{\rho}^{\mathrm{M} / \mathrm{E}}=\rho\left\{1+\sum_{\boldsymbol{K} \neq 0} \mu_{\boldsymbol{K}^{2}}^{2}(K ; \hat{\rho})+\rho \sum_{\boldsymbol{K} \neq 0} \sum_{\boldsymbol{K}^{\prime} \neq 0,-\boldsymbol{K}} \mu_{\boldsymbol{K}} \mu_{\boldsymbol{K}^{\prime}} \mu_{-\left(\boldsymbol{K}+\boldsymbol{K}^{\prime}\right)}\left[\frac{v\left(\boldsymbol{K}, \boldsymbol{K}^{\prime} ; \hat{\rho}\right)}{N}\right]\right\}$.

As in equation (5) the three-particle term only appears in the EMA.

Since a free minimization of the approximate Helmholtz free energy with respect to an infinite number of Fourier components $\mu_{\boldsymbol{K}}$ at all RLV's is intractable, we make 
a simple ansatz for the one-particle density which is a superposition of normalized Gaussians centered around the lattice sites of the triangular lattice:

$$
\rho(\boldsymbol{r})=\frac{n_{c} \alpha}{\pi} \sum_{\boldsymbol{R}} \exp \left[-\alpha|\boldsymbol{r}-\boldsymbol{R}|^{2}\right],
$$

where $\alpha$ is the localization strength, $n_{c}$ is the average number of particles occupying a lattice site, yielding a vacancy concentration $n_{v}=1-n_{c}$, and $\{\boldsymbol{R}\}$ is the set of Bravais lattice vectors of the triangular lattice with lattice constant $a=\left(\sqrt{3} n_{c} / 2 \rho\right)^{1 / 2}$. Thus, the Fourier components $\mu_{\boldsymbol{K}}$ now simply read

$$
\mu_{\boldsymbol{K}}=e^{-\boldsymbol{K}^{2} / 4 \alpha} .
$$

The ansatz, equation (13), was chosen in such a way that the system forms a triangular lattice for any finite $\alpha$ keeping its average density $\rho$ fixed. For $\alpha \rightarrow 0$ the density profile becomes flat and the system turns into a liquid. We thus end up with two minimization parameters $\alpha$ and $n_{c}$.

This ansatz disregards a possible partition of the system into coexisting liquid and crystal phases of different densities keeping the overall average density fixed. However, this is accounted for by performing a common-tangent construction to the crystal and liquid volume free energy densities in the end. Furthermore, equation (13) disregards the spatial anisotropy of the density site profile at each lattice site. We will see in section 5.3 . where we relax the constraint on the density peaks, that both, the assumption of isotropy and the Gaussian shape are well justified close to the positions of the Bravais lattice vectors, i.e., where the density is reasonably large $(\rho(r) \gtrsim \rho)$.

Employing the ansatz of equation (13) for the inhomogeneous density, the ideal part of the Helmholtz free energy [equation (3)] can now be written as a function of $\alpha$ and $n_{c}$ only: $F_{\text {id }}[\rho(\boldsymbol{r})]=F_{\text {id }}\left(\alpha, n_{c} ; \rho\right)$. For $n_{c}=1$ it reads

$$
\begin{aligned}
& \frac{\beta}{N} F_{\text {id }}\left(\alpha, n_{c}=1 ; \rho\right)=\mathrm{const}+\ln \left(\rho L^{2}\right)+G\left(\alpha^{*}\right), \\
& G\left(\alpha^{*}\right)=\int_{A_{1}} \mathrm{~d} \boldsymbol{x} \frac{\rho\left(\boldsymbol{x}, \alpha^{*}, n_{c}=1\right)}{\rho} \ln \left[\frac{\rho\left(\boldsymbol{x}, \alpha^{*}, n_{c}=1\right)}{\rho}\right],
\end{aligned}
$$

where const is an irrelevant constant and $L$ is a density-independent length scale of the system. $\quad \boldsymbol{x}=\boldsymbol{r} \rho^{1 / 2}$ and $\alpha^{*}=\alpha / \rho$ are the dimensionless space coordinate and localization strength, respectively, and the integral is performed over the area $A_{1}$ of a unit cell. The function $G\left(\alpha^{*}\right)$ is approximated for small and large localization strengths by its analytically known asymptotics

$$
G\left(\alpha^{*}\right) \simeq \begin{cases}G_{1}\left(\alpha^{*}\right)=\sum_{\boldsymbol{K}_{*} \neq 0} \exp \left[-\boldsymbol{K}_{*}^{2} / 2 \alpha^{*}\right], & \alpha^{*} \ll 1 \\ G_{2}\left(\alpha^{*}\right)=\ln \left(\alpha^{*} / \pi\right)-1, & \alpha^{*} \gg 1,\end{cases}
$$

where $\boldsymbol{K}_{*}=\boldsymbol{K} / \rho$ are the dimensionless RLV's. For intermediate values of $2 \leq \alpha^{*} \leq 50$ the function $G\left(\alpha^{*}\right)$ was calculated numerically. The function $G\left(\alpha^{*}\right)$ and the asymptotics of equation (17) are plotted as a function of $\alpha^{*}$ in figure 1 .

The ideal free energy for values $n_{c} \neq 1$ is obtained via the simple scaling relation

$$
\frac{\beta}{N} F_{\text {id }}\left(\alpha, n_{c}, \rho\right)=\text { const }+\ln \left(\rho L^{2}\right)+G\left(n_{c} \alpha^{*}\right) \text {. }
$$




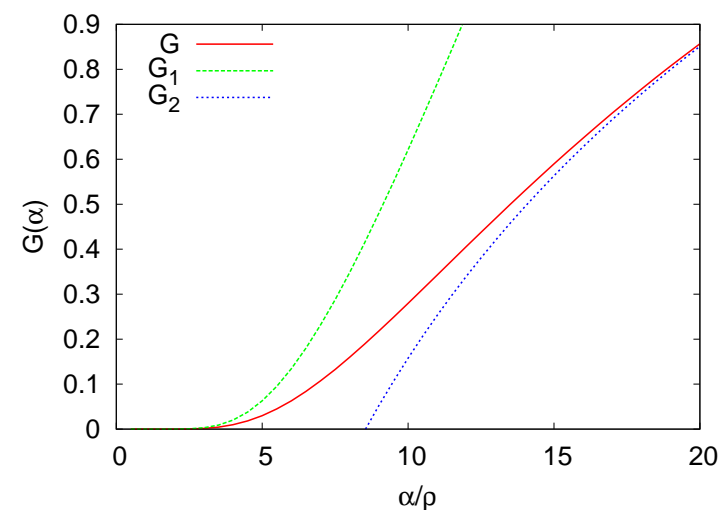

Figure 1. The function $G(\alpha / \rho)$ and its analytically known asymptotics for small and large localization strength.

\section{The dipolar system}

We now turn to the system of monodisperse particles which repel each other with an inverse-power pair potential $u(r)=u_{0} / r^{3}$, where $u_{0}$ is a parameter with dimensions of energy $\times$ volume. For the specific realization of two-dimensional paramagnetic colloids of susceptibility $\chi$ exposed to a magnetic field $\boldsymbol{B}$ which is directed perpendicular to the 2D plane, we have $u_{0}=(\chi \boldsymbol{B})^{2} / 2$ in Gaussian units [30]. Here, we assume perfect alignment of the magnetic dipoles with the external field which is well justified for $\chi B^{2} \gg k_{B} T$ [31]. The thermodynamics and structure depend, due to simple scaling, only on one relevant dimensionless coupling parameter [32]

$$
\Gamma=\frac{u_{0} \rho^{3 / 2}}{k_{B} T} .
$$

Therefore, it is convenient to express all quantities in terms of $\Gamma$ and consider coupling parameters rather than densities via this scaling relation. Correspondingly, the excess free energy within the SOT and TOT [equation 1 now read

$$
\begin{aligned}
\beta F_{\mathrm{ex}}^{\mathrm{S} / \mathrm{T}}(\Gamma, \alpha) / N & =\beta f_{0}(\Gamma)-\frac{1}{2} \sum_{\boldsymbol{K} \neq 0} e^{-K^{2} / 4 \alpha} \hat{c}_{0}^{(2)}(K ; \Gamma) \\
& -\frac{1}{6} \sum_{\boldsymbol{K} \neq 0} \sum_{\boldsymbol{K}^{\prime} \neq 0,-\boldsymbol{K}} e^{-\left(K^{2}+K^{\prime 2}+\left(\boldsymbol{K}+\boldsymbol{K}^{\prime}\right)^{2}\right) / 4 \alpha} \hat{c}_{0}^{(3)}\left(\boldsymbol{K}, \boldsymbol{K}^{\prime} ; \Gamma\right),
\end{aligned}
$$

the third term only appearing in the TOT. Here, $\Gamma$ is the coupling constant corresponding to the average density $\rho$ according to equation $(19), \hat{c}_{0}^{(2)}=\rho \tilde{c}_{0}^{(2)}$, and $\hat{c}_{0}^{(3)}=\rho^{2} \tilde{c}_{0}^{(3)}$ are the dimensionless correlation functions of the fluid in reciprocal space, respectively.

For the MWDA and EMA, the weighted coupling constants $\hat{\Gamma}$ now read $\hat{\Gamma}(\Gamma, \alpha)=\Gamma\left[1-\frac{1}{3 \beta \hat{\Gamma} f_{0}^{\prime}(\hat{\Gamma})} \sum_{K \neq 0} e^{-K^{2} / 2 \alpha} \hat{c}_{0}^{(2)}(K ; \hat{\Gamma})\right.$ 


$$
\left.-\frac{\Gamma^{2 / 3}}{9 \beta \hat{\Gamma}^{5 / 3} f_{0}^{\prime}(\hat{\Gamma})} \sum_{\boldsymbol{K} \neq 0} \sum_{\boldsymbol{K}^{\prime} \neq 0,-\boldsymbol{K}} e^{-\left(K^{2}+K^{\prime 2}+\boldsymbol{K} \boldsymbol{K}^{\prime}\right) / 2 \alpha} \hat{c}_{0}^{(3)}\left(\boldsymbol{K}, \boldsymbol{K}^{\prime} ; \hat{\Gamma}\right)\right]^{3 / 2},
$$

where $f_{0}^{\prime}(\Gamma)$ is the derivative of the excess free energy density with respect to coupling constant. As in equation (12) the third term only appears in the EMA.

In order to solve equations (20) and (21) we need the two- and three-particle correlation functions $\hat{c}_{0}^{(2)}(k ; \Gamma)$ and $\hat{c}_{0}^{(3)}(k ; \Gamma)$ and the excess free energy density $f_{0}(\Gamma)$ of the corresponding liquid for a wide range of coupling constants $\Gamma$. The two-particle correlation function is obtained with liquid state integral equation theory or from computer simulations. In the first case, following the procedure described in Ref. [33] we solve the Ornstein-Zernicke (OZ) equation 34]

$$
\hat{h}(k)=\frac{\hat{c}_{0}^{(2)}(k)}{1-\hat{c}_{0}^{(2)}(k)},
$$

which relates the dimensionless Fourier transform $\hat{h}(k)=\rho \tilde{h}(k)$ of the total correlation function $h(r)$ to the direct pair correlation function $\hat{c}_{0}^{(2)}(k)$, numerically. Note that the density has been absorbed in both the Fourier transform of the total correlation function $\hat{h}(k)$ and in the direct correlation function $\hat{c}_{0}^{(2)}(k)$. The total correlation function is connected to the pair distribution function via $g(r)=h(r)+1$.

The solution of equation 222 for the two unknown quantities $\hat{h}(k)$ and $\hat{c}_{0}^{(2)}(k)$ demands a constitutive equation, the so called closure relation which for any nontrivial case can only be determined approximatively. Two approaches which proved successful for the description of fluids with long-range interactions will be applied here, the hypernetted chain (HNC) [34] and the Rogers-Young (RY) closure relation [35]. They can both be written as

$$
h(r)=e^{-\beta u(r)}\left\{1+f(r)^{-1}\left(e^{\chi(r) f(r)}-1\right)\right\}-1,
$$

where $\chi(r)=h(r)-c_{0}^{(2)}(r)$ is the indirect correlation function. $f(r)=1-e^{-\xi r}$ is a 'mixing function' with an adjustable parameter $0 \leq \xi \leq \infty$ which is either sent to infinity (HNC) - which is equivalent to letting $f(r) \rightarrow 1$ - or chosen to guarantee thermodynamic consistency between virial and compressibility route to the free energy (RY).

The coupled equations (22) and (23) are iteratively solved by applying the method of fast Fourier transforms for radially symmetric two-dimensional problems as suggested by Caillol et al [36] and as also summarized in appendix A of [33]. In order to reach rapid convergence an iteration procedure for the indirect correlation function $\chi(r)$ is used, since its Fourier transform, $\tilde{\chi}(k)$, decays more rapidly with increasing $k$ than $\tilde{h}(k)$. The iteration scheme now consists of making an ansatz for $c_{0}^{(2)}$, calculating $\chi$ according to equation 22, obtaining the next estimate of $c_{0}^{(2)}$ via equation 23), inserting this into equation (22), etc., until convergence is obtained.

Applying this procedure we are able to calculate $\hat{c}_{0}^{(2)}(k ; \Gamma)$ for coupling constants $\Gamma$ much larger than the experimentally known coupling of freezing $\Gamma_{f} \approx 10[26]$ 


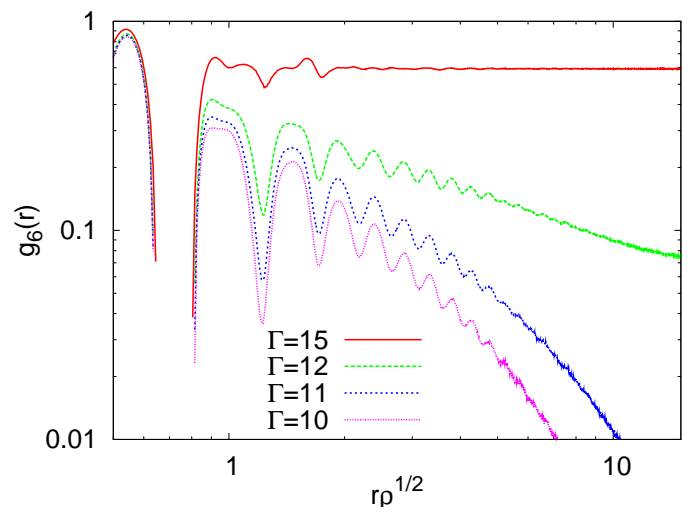

Figure 2. The bond-orientational order parameter $g_{6}(\boldsymbol{r})$ for different coupling constants $\Gamma$ as obtained by computer simulation. $g_{6}$ decays exponentially for coupling constants $\Gamma \lesssim 11$ indicating the system to be in the fluid state.

which enables us to calculate the Helmholtz free energy of the system deep inside the thermodynamically stable crystalline region.

More accurate pair correlation functions can be obtained from computer simulations. We have performed extensive Monte Carlo computer simulations [37] in a quadratic simulation box of size $L \times L$ comprising 900 particles employing periodic boundary conditions in order to measure the pair distribution function $g_{\mathrm{s}}(r)=h_{\mathrm{s}}(r)+1$, the subscript ' $\mathrm{s}$ ' denoting the simulation result. Since the accessible range of $h_{\mathrm{s}}(r)$ is limited to distances $r$ smaller than a cutoff radius $r_{c} \lesssim L / 2$ we employed an extrapolation technique suggested by Verlet [38] to obtain the complete pair correlation function: Verlet defined a closure relation to the Ornstein-Zernicke equation

$$
\begin{aligned}
& h(r)=h_{\mathrm{s}}(r) \quad r<r_{c} \\
& c_{0}^{(2)}(r)=c_{\mathrm{HNC}}^{(2)}(r) \quad r>r_{c},
\end{aligned}
$$

where $c_{\mathrm{HNC}}(r)$ is given in equation (23). The Verlet closure relation [equation (24)] together with the Ornstein-Zernicke equation [equation (22)] uniquely specify the direct correlation function $c_{0}^{(2)}(r)$ for all radii $r$ and thus also yield the correlation function in reciprocal space $\hat{c}_{0}^{(2)}(k)$. As for the HNC and the RY closures the Ornstein-Zernicke equation and the Verlet closure were solved iteratively via the indirect correlation function $\chi$. Furthermore, $r_{c}$ was chosen the largest root of $h(r)$ still smaller than $L / 2$.

For the Verlet data we checked that the simulated system does not crystallize for coupling constants $\Gamma \lesssim 11$. Here, the freezing-criterion was chosen a non-exponential decay of the bond-orientational order parameter $g_{6}(\boldsymbol{r})=\left\langle\exp \left[i 6\left[\theta(\boldsymbol{r})-\theta\left(\boldsymbol{r}^{\prime}\right)\right]\right]\right\rangle$, where $\theta(\boldsymbol{r})$ is the angle of the bond connecting two neighboring particles according to the Voronoi construction (see figure 2). The application of the Verlet closure within the DFT formalism was thus restricted to the range $0 \leq \hat{\Gamma} \lesssim 11$.

The Fourier transforms $\hat{c}_{0}^{(2)}(k)$ of the two-particle direct correlation functions obtained from the three different closure relations (HNC, RY, Verlet) are shown in figure 3 for $\Gamma=9$, which is close to the experimentally determined coupling constant 


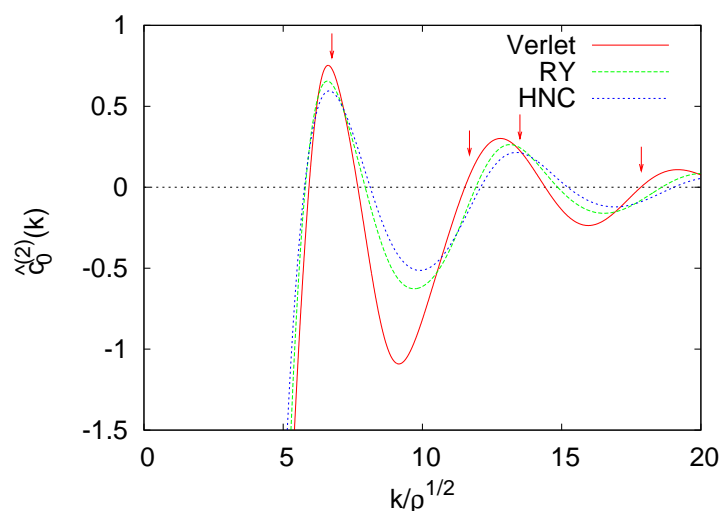

Figure 3. The dimensionless Fourier transform $\hat{c}_{0}^{(2)}(k)$ of the two-particle direct correlation function at $\Gamma=9$, plotted against $k / \rho^{1 / 2}$. Shown are simulation data using the Verlet closure, liquid integral equation theory using the RY closure, and liquid integral equation theory using the HNC closure. The arrows indicate the positions of the first four reciprocal lattice vectors of the triangular lattice.

of freezing $\Gamma_{f} \simeq 10$ [26]. The HNC closure underestimates the pair structure strongly while the RY closure is closer to the simulation data. We also show the positions of the first four reciprocal lattice vectors of the triangular lattice with lattice constant $a=(\sqrt{3} / 2 \rho)^{1 / 2}$. The value of $\hat{c}_{0}^{(2)}$ at these lattice vectors crucially influences the solid free energies, as can be seen from equations 20) and 21).

Within the RY-approach the excess free energy density $f_{0}$ is obtained by integrating the compressibility which is inversely proportional to the static structure factor:

$$
\beta f_{0}(\Gamma)=\frac{2}{3} \int_{0}^{\Gamma} \frac{\mathrm{d} \Gamma^{\prime}}{\Gamma^{\prime}}\left[\frac{\beta P}{\rho}-1\right],
$$

where the pressure $P$ is given by

$$
\frac{\beta P}{\rho}-1=\frac{2}{3} \int_{0}^{\Gamma} \frac{\mathrm{d} \Gamma^{\prime}}{\Gamma^{\prime 1 / 3}}\left[1-\hat{c}_{0}^{(2)}\left(k=0 ; \Gamma^{\prime}\right)\right] .
$$

In order to obtain the excess free energy density from the simulation data we make use of the relation [39]

$$
\beta\left\langle u_{\mathrm{ex}}\right\rangle=\beta \frac{\partial \beta f_{0}}{\partial \beta}=\Gamma \frac{\partial \beta f_{0}}{\partial \Gamma}
$$

between the average excess energy density $\left\langle u_{\mathrm{ex}}\right\rangle=\frac{1}{2}\left\langle\Sigma_{i \neq j} u_{i, j}\right\rangle$ and $f_{0}$ and integrate the former. Note that for both of our approaches, the RY and the Verlet closure, the virial and the compressibility route are equivalent. As the energy dominates the free energy in the strong coupling limit, $\Gamma \gtrsim 1$, the excess free energy density scales roughly linearly with coupling constant, as can be seen from figure 4.

For the EMA we need the three-particle correlation function $\tilde{c}_{0}^{(3)}\left(\boldsymbol{k}, \boldsymbol{k}^{\prime} ; \rho\right)$ of the underlying fluid for a wide range of coupling constants. We use here two conceptually different approximations: The first approximation is by Denton and Ashcroft [24] (DA) which is based on a weighted density approximation to the first order direct correlation 


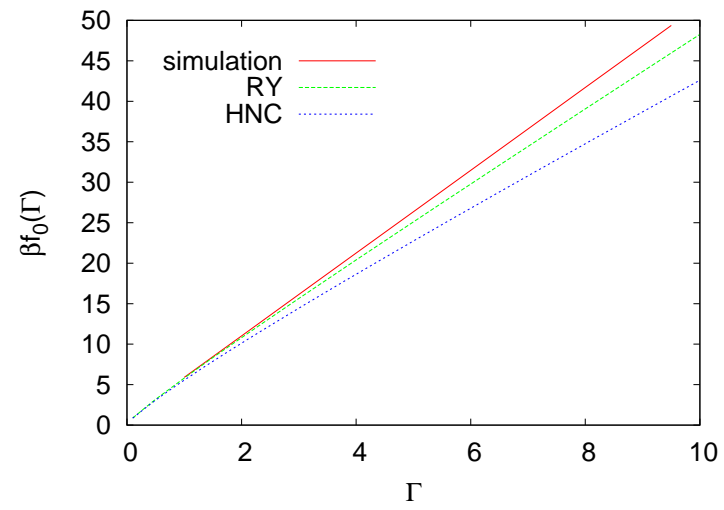

Figure 4. The excess free energy density $\beta f_{0}(\Gamma)$ as a function of coupling constant $\Gamma$ using the Verlet closure, the RY closure and the HNC closure.

function $c^{(1)}(\boldsymbol{r} ; \rho(\boldsymbol{r}))$ of an inhomogeneous system. The DA approach leads to an analytic expression of $\tilde{c}_{0}^{(3)}$ in terms of the one- and two-particle correlation functions $c_{0}^{(1)}, \tilde{c}_{0}^{(2)}$ of the liquid and their derivatives with respect to density:

$\tilde{c}_{0}^{(3) \mathrm{DA}}\left(\boldsymbol{k}, \boldsymbol{k}^{\prime}\right)=\frac{1}{3}\left[\tilde{f}^{\mathrm{DA}}\left(|\boldsymbol{k}|,\left|\boldsymbol{k}^{\prime}\right|\right)+\tilde{f}^{\mathrm{DA}}\left(|\boldsymbol{k}|,\left|\boldsymbol{k}+\boldsymbol{k}^{\prime}\right|\right)+\tilde{f}^{\mathrm{DA}}\left(\left|\boldsymbol{k}^{\prime}\right|,\left|\boldsymbol{k}+\boldsymbol{k}^{\prime}\right|\right)\right]$,

where

$\tilde{f}^{\mathrm{DA}}\left(k, k^{\prime}\right)=\frac{1}{c_{0}^{(1) \prime}}\left[\tilde{c}_{0}^{(2)}(k) \tilde{c}_{0}^{(2) \prime}\left(k^{\prime}\right)+\tilde{c}_{0}^{(2) \prime}(k) \tilde{c}_{0}^{(2)}\left(k^{\prime}\right)\right]-\frac{c_{0}^{(1) \prime \prime}}{\left[c_{0}^{(1) \prime}\right]^{2}} \tilde{c}_{0}^{(2)}(k) \tilde{c}_{0}^{(2)}\left(k^{\prime}\right)$.

Here, primes denote derivatives with respect to density, as above. The DA approximation - by construction - fulfills the symmetry condition

$$
\tilde{c}_{0}^{(3) \mathrm{DA}}\left(\boldsymbol{k}, \boldsymbol{k}^{\prime}\right)=\tilde{c}_{0}^{(3) \mathrm{DA}}\left(\boldsymbol{k}, \boldsymbol{k}+\boldsymbol{k}^{\prime}\right)=\tilde{c}_{0}^{(3) \mathrm{DA}}\left(\boldsymbol{k}^{\prime}, \boldsymbol{k}+\boldsymbol{k}^{\prime}\right) .
$$

The derivatives $\tilde{c}_{0}^{(2) \prime}(k)$ were obtained by applying a simple finite difference method bearing in mind that

$\rho^{2} \tilde{c}_{0}^{(2) \prime}(k ; \rho)=\frac{1}{2}\left[3 \Gamma \frac{\partial \hat{c}_{0}^{(2)}\left(k \rho^{-1 / 2} ; \Gamma\right)}{\partial \Gamma}-k \rho^{-1 / 2} \frac{\partial \hat{c}_{0}^{(2)}\left(k \rho^{-1 / 2} ; \Gamma\right)}{\partial k \rho^{-1 / 2}}-2 \hat{c}_{0}^{(2)}\left(k \rho^{-1 / 2} ; \Gamma\right)\right]$.

We calculated $\tilde{c}_{0}^{(3) \mathrm{DA}}\left(\boldsymbol{k}, \boldsymbol{k}^{\prime}\right)$ taking the direct correlation function from both the RY and the Verlet closure. As pointed out in Refs. [22, 24, 40] the DA model, although itself not derived from a free energy functional but from an approximate one-particle correlation function, is very similar to different approaches, all based on taking three successive functional derivatives of approximate free energy functionals.

We also employed another approximation for $c_{0}^{(3)}$, namely a factorization ansatz of Barrat, Hansen and Pastore (BHP) [23]. The approximation reads

$$
c_{\mathrm{BHP}}^{(3)}\left(\boldsymbol{r}, \boldsymbol{r}^{\prime}\right)=t(r) t\left(r^{\prime}\right) t\left(\left|\boldsymbol{r}-\boldsymbol{r}^{\prime}\right|\right) .
$$


The function $t(r)$ can be uniquely determined from the second of the sum rules in equation (9) which in $r$-space now reads

$$
\int \mathrm{d} \boldsymbol{r}^{\prime} c_{0}^{(3)}\left(\boldsymbol{r}, \boldsymbol{r}^{\prime} ; \rho\right)=\int \mathrm{d} \boldsymbol{r}^{\prime} t(r) t\left(r^{\prime}\right) t\left(\left|\boldsymbol{r}-\boldsymbol{r}^{\prime}\right|\right)=\frac{\partial c_{0}^{(2)}(r ; \rho)}{\partial \rho} .
$$

We solved equation (33) numerically for $t(r)$ applying the method of 'steepest descent' as outlined in appendix B of reference [23]. As opposed to the simple finite difference approach above the derivatives $c_{0}^{(2) \prime}(k)$ were now obtained by iteratively solving the coupled differentiated Ornstein-Zernicke equation and the differentiated RY closure relation, as outlined in appendix B of [23]. Since it proved difficult to reach convergence of the iteration procedure we did not pursue this method using the Verlet closure. The triplet-correlation function was then obtained by a double Fourier transform of equation (33) using a standard expansion in Legendre polynomials, as outlined in appendix A of [23].

In the single summation in equation (21) we consider all RLV's of absolute value $|\boldsymbol{K}| \leq 33\left|\boldsymbol{K}_{1}\right|$, where $\boldsymbol{K}_{1}$ is the smallest RLV of the triangular lattice - this comprises the first 299 stars of RLV's, which is by far sufficient to reach convergence of the single summation. The double summation is performed over sets of equivalent triangles of RLV's which are each characterized by the absolute values of the two RLV's $\boldsymbol{K}$ and $\boldsymbol{K}^{\prime}$, and by the absolute value of there included angle. For the DA model and for the BHP model we include 42 sets of triangles of RLV's, where that RLV of the three RLV's, $\boldsymbol{K}, \boldsymbol{K}^{\prime}, \boldsymbol{K}-\boldsymbol{K}^{\prime}$, with the largest absolute value satisfies $|\boldsymbol{K}| \leq 4\left|\boldsymbol{K}_{1}\right|$, which also guarantees convergence of the double sum.

\section{Results}

We first study the influence of the explicit inclusion of the triplet correlation functions obtained with the DA model and with the BHP model on the approximate excess free energy according to the TOT as compared to the simpler SOT, and according to the EMA as compared to the MWDA, respectively. For all six approaches we use the two different closure relations of Rogers and Young [equation (23)], and of Verlet [equation (24)], respectively.

\subsection{Gaussian profiles, no vacancies}

In order to keep things simple in the beginning we keep the number of particles occupying a lattice site, $n_{c}$, in equation (13) fixed (i.e., $n_{c}=1$ ) and thus end up with a single order parameter, the dimensionless localization strength $\alpha^{*} \equiv \alpha / \rho$.

In figure $5(a, b)$ we show the weighted coupling constant and the associated excess free energy difference per particle between the solid and the liquid state $F_{\text {ex }}\left(\alpha^{*}\right) / N-f_{0}$, according to equation (4), as functions of localization strength $\alpha^{*}$ for a value of $\Gamma=9$ which is close to the experimentally known value of freezing, $\Gamma_{f} \simeq 10$ [26], for the MWDA and for the EMA, using the RY or the Verlet approach to the direct correlation 

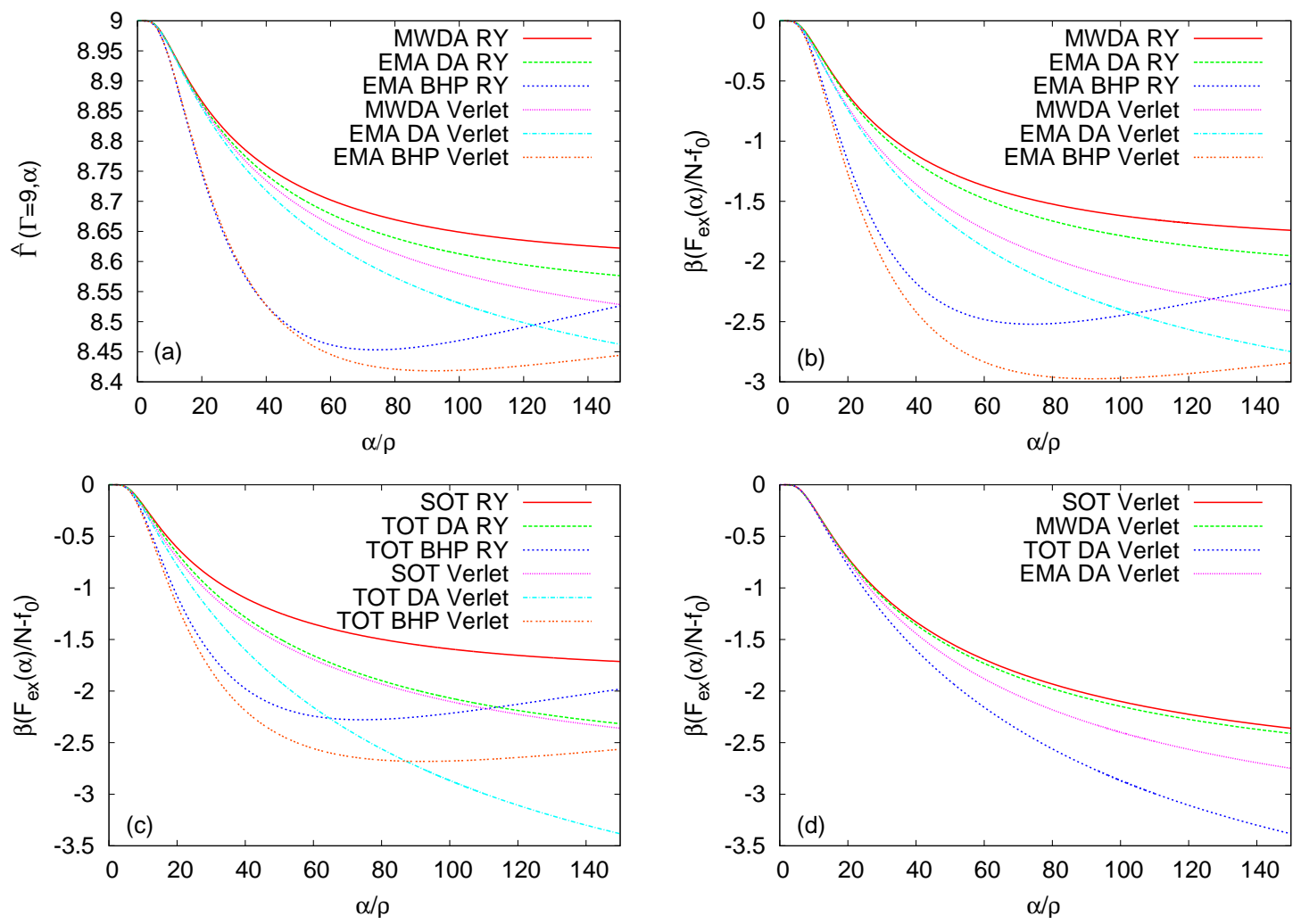

Figure 5. (a) The weighted coupling constant as a function of $\alpha^{*}$ within the MWDA and within the EMA using $c_{0}^{(2)}$ from the RY and from the Verlet closure, and using $c_{0}^{(3)}$ from the DA and the BHP model for $\Gamma=9$. (b) The approximate excess free energy difference per particle $f_{0}\left(\hat{\Gamma}\left(\alpha^{*}\right)\right)-f_{0}(\Gamma)$ as a function of $\alpha^{*}$ for the same approximations as in (a). (c) The approximate excess free energy difference per particle $F_{\text {ex }}\left(\alpha^{*}\right) / N-f_{0}\left(\alpha^{*}=0\right)$ as a function of $\alpha^{*}$ obtained within the SOT and TOT using the same approximations for the two- and three-particle correlation functions as in (a,b) for $\Gamma=9$. (d) Comparison of $F_{\text {ex }}$ obtained within the four different approximate theories MWDA, EMA, SOT, and TOT using $c_{0}^{(2)}$ from the Verlet closure, and using $c_{0}^{(3)}$ from the DA model for $\Gamma=9$.

function and using the two different approaches for the triplet correlation function, the DA and the BHP model. The latter are both based on the direct correlation functions used for the respective two-particle term. In figure 5(c) the excess free energy for the simpler SOT and TOT are plotted as a function of $\alpha^{*}$ for the same approximations to the correlation functions. In figure 5(d) the non-perturbative and the perturbative approaches are compared. Different interesting features of the different approximations are observed:

(i) For all approaches used except for those where $c_{0}^{(3)}$ is obtained within the BHP model the excess free energy decreases monotonically with increasing localization strength $\alpha^{*}$, reaching a plateau for $\alpha^{*} \approx 400$ [c.f. figure 5(b,c)]. However, employing the BHP model to the triplet-correlation function leads to an increase of $\hat{\Gamma}\left(\alpha^{*}\right)$ and $F_{\text {ex }}\left(\alpha^{*}\right)$ for values of $\alpha^{*} \gtrsim 80$. The former behavior is intuitively expected and has also been 

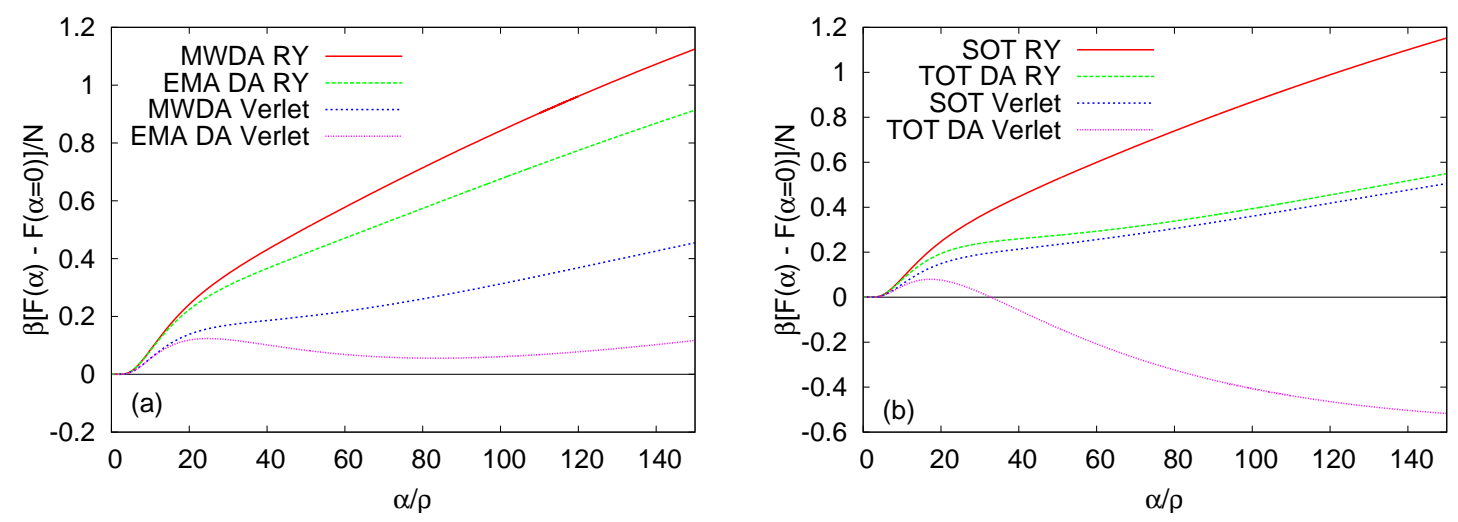

Figure 6. The total free energy difference per particle $\Delta F(\Gamma) / N$ as a function of $\alpha^{*}$ within the MWDA and EMA (a), and within the SOT and TOT (b) using $c_{0}^{(2)}$ from the RY and from the Verlet closure, and using $c_{0}^{(3)}$ from the DA model for $\Gamma=9$.

observed in the original MWDA [21]-localization is favoured by the excess part of the free energy. Once the density peaks become very narrow, a further increase of $\alpha^{*}$ does not change the excess free energy further. On the other hand, the rise of $\hat{\Gamma}$ and of $F_{\text {ex }}$ within the BHP model is regarded as unphysical. We therefore do not consider the BHP model any further.

(ii) Both within the DA model and within the BHP model (for $\alpha^{*} \lesssim 80$ ) the sign of the third term in equation 21) is negative, i.e., the value of $\hat{\Gamma}$ is decreased as compared to the pure MWDA and thus freezing is favoured. It is also interesting to note, that within the DA model the triplet-part in equation (21) is much smaller than the secondorder term while it is significantly larger within the BHP model. This same behavior had already been found for hard spheres in three dimensions [22].

(iii) Although the direct correlation functions using the RY-and the Verlet-closures do not differ by more than $\sim 10 \%$ at the position of the most important first RLV (cf. figure 4 the difference in $\hat{\Gamma}$ between the two is quite pronounced which is due to the self-consistency relation in equation (21). Furthermore, as shown in figure 5(b) the difference in excess free energy is even more enhanced.

(iv) Inclusion of higher than second-order terms in a non-perturbative way within the MWDA reduces the excess free energy as compared to the simpler SOT [c.f. figure $5(\mathrm{~d})$. However, inclusion of higher than third-order terms within the EMA increases the excess free energy with respect to the TOT.

The total Helmholtz free energy per particle is obtained by adding to the excess part $F_{\text {ex }}$ the ideal part $F_{\text {id }}$ according to equation (2). The free energy difference per particle $\Delta F / N=\left[F\left(\alpha^{*} ; \Gamma\right)-F\left(\alpha^{*}=0 ; \Gamma\right)\right] / N$ is plotted in figure 6 as a function of $\alpha^{*}$, for the same value of $\Gamma=9$ as in figure 5 for the SOT and TOT [figure6(a)], and for the MWDA and EMA [figure 6(b)], respectively, using $c_{0}^{(2)}$ from the RY and from the Verlet closure, and using $c_{0}^{(3)}$ obtained within the DA model. It is found that the different curves of $\Delta F / N$ show qualitatively very different behavior for the coupling of $\Gamma=9$ : 


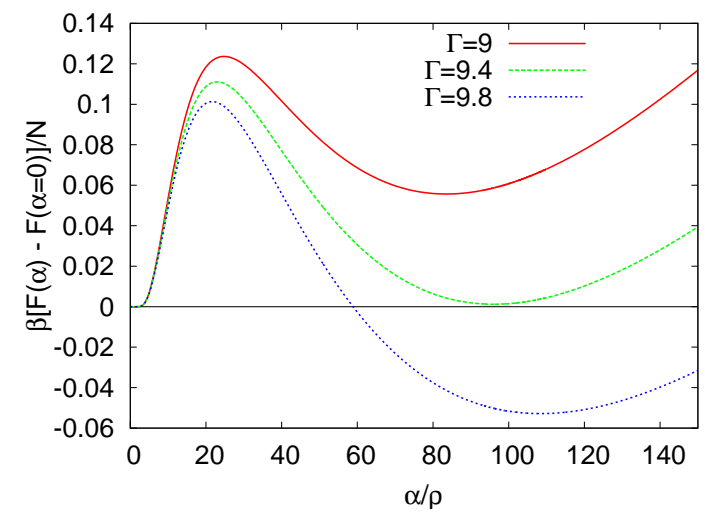

Figure 7. The total free energy difference per particle $\Delta F(\Gamma) / N$ as a function of $\alpha^{*}$ within the EMA using $c_{0}^{(2)}$ from the Verlet closure, and using $c_{0}^{(3)}$ from the DA model for $\Gamma=9,9.4,9.8$.

While the free energy increases monotonically with $\alpha^{*}$ within the SOT and MWDA and within the TOT and EMA employing the RY closure it displays a local minimum with respect to $\alpha^{*}$ at a finite value of $\alpha^{*}$ within the EMA and TOT, employing the Verlet closure, this local minimum even turning the deep global minimum within the TOT at $\alpha^{*} \approx 213$. The appearance of a global minimum at a finite value of $\alpha^{*}$ corresponds to a thermodynamically stable crystalline state while the global minimum at $\alpha^{*}=0$ indicates a stable fluid system.

In figure 7, we display the total free energy obtained within the EMA employing the DA model with the Verlet closure for three different values of $\Gamma=9.0,9.4,9.8$. We thus conclude from figure 7 this has already been presented in Ref. [17] that the EMA employing the Verlet closure and the DA model yields a transition from the fluid to the solid close to $\Gamma=9.4$ : while for $\Gamma=9.0$ the fluid is stable as indicated by the minimal value at $\alpha^{*}=0$, fluid-solid coexistence is achieved at $\Gamma=9.4$ (see the two equal minima in figure 7). The solid phase, on the other hand, is clearly stable for $\Gamma=9.8$. The localization parameter at coexistence is roughly $\alpha_{\text {min }}^{*} \approx 100$.

The curves always displays a local minimum with respect to $\alpha^{*}$ at $\alpha^{*}=0$. This is in accordance with the mean-field character of any approximation to the DFT, which ignore fluctuations leading to a breakdown of long-range order in one and two dimensions. Therefore, a first-order transition between fluid and solid state is always predicted, i.e., the liquid system always has to overcome a free energy barrier in order to reach the thermodynamically stable crystalline state.

The freezing and melting transition constants for the first-order phase transition predicted by the different approximations to the DFT, $\Gamma_{s}$ and $\Gamma_{f}$, respectively, are obtained by using Maxwell's double tangent construction to the fluid and crystal volume free energy densities $\Gamma^{2 / 3} F / N \propto F / V$, where $F$ denotes the minimum free energy with respect to $\alpha$, and $\Gamma^{2 / 3}$ is proportional to the average density $\rho$ of the system [c.f. equation (19)]. $\Gamma_{s}$ and $\Gamma_{f}$ correspond to the freezing and melting densities, $\rho_{s}$ and 


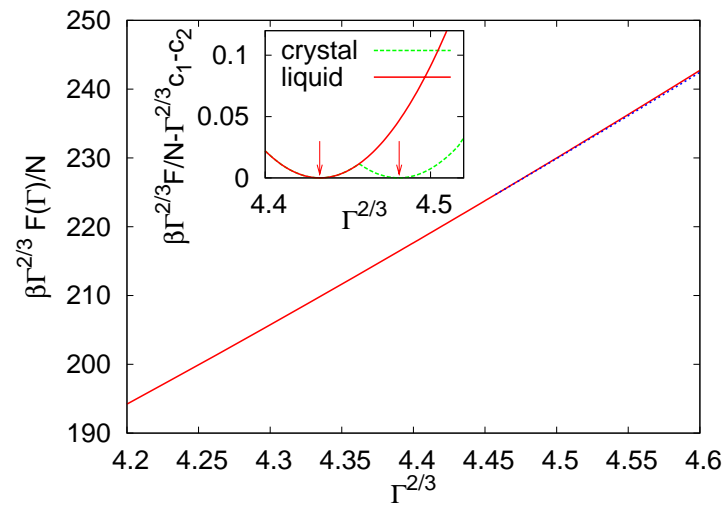

Figure 8. The liquid (solid line) and crystal (dotted line) volume free energy densities $\Gamma^{2 / 3} F / N$ obtained within the EMA using the Verlet closure and the DA model as a function of $\Gamma^{2 / 3}$. The inset show the tilted free energy densities around the transition values $\Gamma_{s}, \Gamma_{f}$, as indicated by arrows.

Table 1. Freezing and melting parameters $\Gamma_{f}$ and $\Gamma_{s}$, the widths of the coexistence regions $\Delta \Gamma=\Gamma_{s}-\Gamma_{f}$, the relative displacement parameters $\gamma$, and the pressures $P$ at coexistence obtained within: the SOT with the RY closure (first row); the TOT with the RY closure (second row); the TOT with the Verlet closure (third row); the MWDA with the RY closure (forth row); the EMA with the RY closure (fifth row); the EMA with the Verlet closure (sixth row), where all three-particle correlation functions were obtained with the DA model using the respective pair-correlation function as input. The last row displays experimental parameters for the isotropic-hexatic transition, the hexatic-crystal transition and the Lindemann parameter, obtained from real-space microscopy measurements of magnetic colloids confined to an air-water interface.

\begin{tabular}{l|lllll} 
& $\Gamma_{f}$ & $\Gamma_{s}$ & $\Delta \Gamma$ & $\gamma$ & $\beta P\left(\Gamma_{f}\right) / \rho$ \\
\hline SOT with RY & 42.85 & 42.92 & 0.07 & 0.017 & 288.3 \\
TOT with RY & 13.49 & 13.62 & 0.13 & 0.021 & 93.1 \\
TOT with Verlet & 6.79 & 6.97 & 0.18 & 0.019 & 53.1 \\
MWDA with RY & 41.07 & 41.13 & 0.06 & 0.017 & 276.1 \\
EMA with RY & 23.0 & 23.08 & 0.09 & 0.020 & 156.9 \\
EMA with Verlet & 9.33 & 9.49 & 0.16 & 0.020 & 72.6 \\
Experiment & 10.0 & 10.75 & - & 0.038 & -
\end{tabular}

$\rho_{f}$, respectively. The volume free energy density is exemplarily shown for the EMA using the Verlet closure and the DA model in figure 8. Within this approximation we obtain freezing and melting with a narrow coexistence gap $\Delta \Gamma=\Gamma_{s}-\Gamma_{f}$. Table I summarizes the freezing/melting parameters for all the approximations made. The data are compared against experimental results obtained from real-space microscopy measurements of magnetic colloids confined to an air-water interface. The experiments give freezing with an intermediate hexatic phase. The liquid-solid transition has also been studied using numerical simulation [28, 29] yielding a slightly higher inverse 
transition temperature between 12.0 and 12.25 but these investigations suffer from finite size effects.

As becomes evident from Table I, the SOT, TOT, and MWDA are not quantitatively satisfying theories as they either over- or underestimate the freezing coupling. Note that the overestimation of the freezing coupling within SOT and MWDA are the reason why it is not possible to feed the "exact" pair structure into these theories. At such high coupling, no fluid pair structures are available since the fluid spontaneously crystallizes in the simulation. The EMA, on the other hand, yields results in close agreement with experimental data. The TOT obviously underestimates the freezing coupling by a factor of $\approx 2$.

More detailed, structural information can be extracted from the localization parameter of the coexisting solid. For all approximations used we find localization parameters at freezing in the range $99<\alpha_{\min }^{*}\left(\Gamma_{s}\right)<115$. Strictly speaking, the localization parameter has no counterpart in "real" $2 \mathrm{D}$ systems since the particles are not localized due to long range fluctuations. However, if one relates the particle displacements to that of their nearest neighbor, one can define a finite quantity as

$\gamma=\rho\left\langle\left(\boldsymbol{u}_{i}-\boldsymbol{u}_{i+1}\right)^{2}\right\rangle$, where $\boldsymbol{u}_{i}$ and $\boldsymbol{u}_{i+1}$ are the displacement vectors of neighboring lattice sites. Disregarding nearest-neighbor correlations $\left\langle\boldsymbol{u}_{i} \cdot \boldsymbol{u}_{i+1}\right\rangle, \gamma$ can be estimated. Since the nearest-neighbor correlations $\left\langle\boldsymbol{u}_{i} \cdot \boldsymbol{u}_{i+1}\right\rangle$ are expected to be positive:

$$
\gamma \lesssim 2 \rho\left\langle\boldsymbol{u}_{i}^{2}\right\rangle \approx 2 / \alpha_{\min }^{*}
$$

By this relation, the localization parameter of the coexisting solid gives a prediction for $\gamma$ which is included in Table I. From experiments, $\gamma$ is known to be close to $\cong 0.038$ [26]. This was shown to be in accordance with harmonic lattice theory [41. The EMA yields $\gamma \lesssim 0.020$, i.e. the EMA roughly overestimates the localization of the particles by a factor of $2 . \quad \gamma$ is smaller than the experimental value, contrarily to what was expected from the inequality (34). This shows that there is still a need to improve the theories in order to correctly predict localization properties. A similar overestimation of the localization is also common in weighted density approximations in three spatial dimensions [21].

Another quantity of interest, which is directly connected to the Helmholtz free energy is the pressure at coexistence which is also included in Table I. It is obtained via the equations (25), 26), 27), depending on whether the RY closure or the simulation data were used.

\subsection{Gaussian profiles, allowing for vacancies}

In this subsection, we relax the constraint of zero vacancy concentration, $1-n_{c}=0$, in equation (13) and instead minimize the total free energy with respect to the two parameters $\alpha$ and $n_{c}$, respectively. However, instead of calculating the phase diagram for all approximations to the DFT and to the pair- and triplet-correlation functions, we focus here on the two non-perturbative approaches, the MWDA using the RY closure and the EMA using the Verlet closure and the DA model. In figure 9 we plot the 

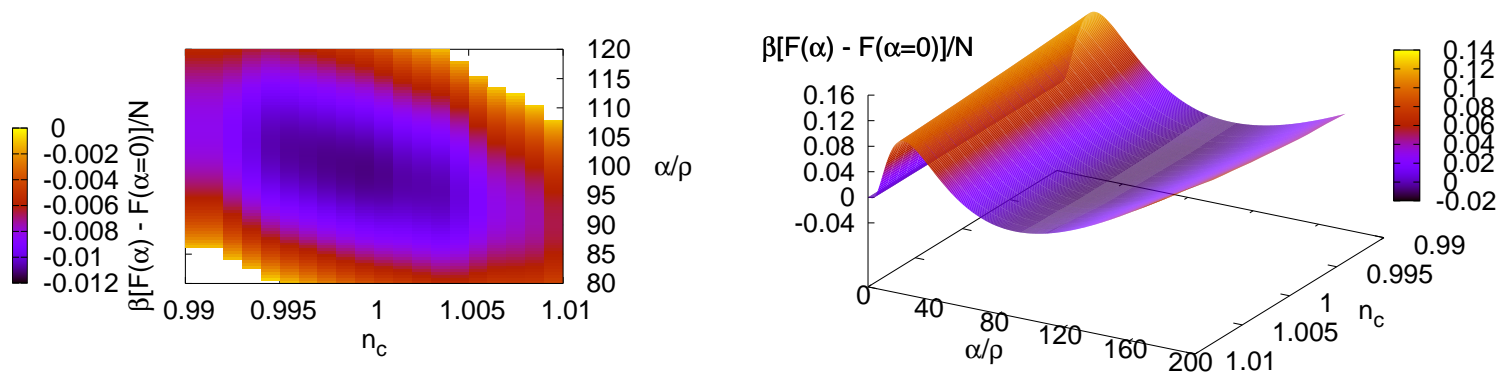

Figure 9. The total free energy difference per particle $\Delta F(\Gamma) / N$ as a function of $\alpha^{*}$ and $n_{c}$ within the EMA using $c_{0}^{(2)}$ from the Verlet closure, and using $c_{0}^{(3)}$ from the DA model for $\Gamma=9.49$. The left panel displays a zoom-in of the right panel.

approximate total free energy per particle of the EMA as a function of $\alpha$ and $n_{c}$ for the freezing coupling constant obtained at fixed $n_{c}=1, \Gamma=9.49$. The minimum of the total free energy is slightly shifted in $n_{c}$ and $\alpha$ from $\left(n_{c} \approx 1, \alpha^{*} \approx 98.7\right)$ towards $\left(n_{c} \approx 0.998, \alpha^{*} \approx 100.5\right)$. As can be seen in figure $9(\mathrm{a})$, the difference in total free energy per particle between the two configurations is only of the order $10^{-4} k_{B} T$, which has no influence on the phase diagram within the accuracy given in Table I.

For the simpler MWDA, however, the vacancy concentration is substantially larger, which has pronounced effects on the phase diagram. In particular, we find the coupling constants of freezing and melting reduced to $\left(\Gamma_{f} \approx 37.35, \Gamma_{s} \approx 37.45\right)$, the liquid being in coexistence with the triangular crystal at the parameters $n_{c} \approx 0.966, \alpha^{*} \approx 200.5$, i.e., the relaxation of $n_{c}$ improves the prediction of the freezing coupling while the Lindemann parameter $\gamma \approx 0.01$ is by a factor of $\approx 2 / 3$ smaller than predicted within the simpler theory keeping $n_{c}=1$ fixed which-compared to the experiment-is worse than the result from the constrained theory.

\subsection{Free minimization}

In this final subsection we completely remove the constraint of Gaussian density peaks. Instead, we minimize the density functional with respect to a free, periodic density field $\rho(x, y)$, which has the periodicity of the hexagonal lattice with lattice constant $a=\left(\sqrt{3} n_{c} / 2 \rho\right)^{1 / 2}$, as above. As laid out in Ref. [42], we minimize the density functional of the SOT with the RY closure with respect to $\rho(\boldsymbol{r})$ by calculating the overdamped relaxation dynamics of a highly ordered hexagonal crystal with the help of dynamical DFT [43, 44, 45, 46] according to

$$
\frac{\partial \rho(\vec{r}, t)}{\partial t}=\beta D \vec{\nabla} \cdot\left(\rho(\vec{r}, t) \vec{\nabla} \frac{\delta F[\rho(\vec{r}, t)]}{\delta \rho(\vec{r}, t)}\right),
$$

where $\beta D$ is the mobility coefficient, which sets the Brownian time scale $\tau_{B}=(\rho D)^{-1}$. Since in this work we are only interested in the equilibrium state reached after long 


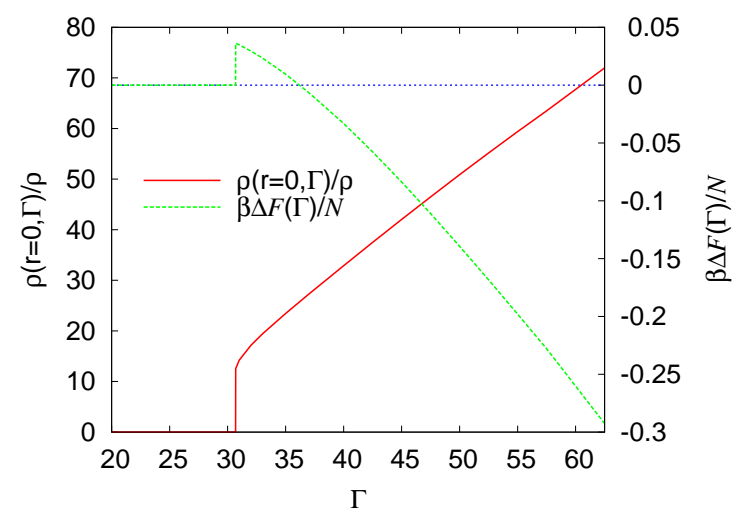

Figure 10. Height of the density peak $\rho(\boldsymbol{r}, \Gamma)$ and difference in free energy $\Delta F(\Gamma)$ as a function of $\Gamma$ obtained from dynamical DFT using the SOT with the RY closure.

time, $\tau_{B}$ is irrelevant in the following considerations, i.e., we use equation (35) just as a minimization procedure to the static DFT. Starting from an initial density profile $\rho(\boldsymbol{r}, t=0)$, equation $(35)$ is solved numerically for times $\left(t / \tau_{B}\right) \lesssim 10$ applying a finite difference method and keeping the coupling constant $\Gamma$ fixed. The maximum time is chosen large enough to guarantee convergence towards a (local) minimum of the free energy landscape. The rectangular periodic box of size $L_{x} \times L_{y}=\sqrt{3} a \times a$ with a discretization of $256 \times 128$ lattice points comprises $2 n_{c}$ particles. Due to lattice symmetry, it suffices to solve the problem in a single elementary cell. For $\rho(\boldsymbol{r}, t=0)$ we choose a superposition of sharply localized Gaussians according to equation (13) with a large localization strength of $\alpha^{*}=200$.

At first, we fix $n_{c}=1$ and calculate the equilibrium density profiles and the according approximate Helmholtz free energies for various coupling constants $0<$ $\Gamma \leq 62.5$. In figure 10 we plot the difference in Helmholtz free energy density $\Delta F(\Gamma) / N=F[\rho(\boldsymbol{r}, t \rightarrow \infty ; \Gamma)] / N-f_{0}(\Gamma)$ between the final (solid/liquid) and the liquid state as a function of $\Gamma$. The system remains crystalline for couplings $\Gamma \gtrsim 30.7$. However, the free energy difference is negative only for $\Gamma \gtrsim 36.2$, which is equivalent with thermodynamic stability. As for the Gaussian parametrization coexistence is found in a narrow gap around $\Gamma \approx 36.2$ which we do not specify here.

In figure 11(a) we plot the equilibrium density profile $\rho(\boldsymbol{r} ; \Gamma)$ for $\Gamma=36$ which is close to freezing. In figure 11(b) the quantity $r^{2} \rho(\boldsymbol{r})$, where $r$ is the distance from a lattice vector, is shown along the two directions [11] and [10], corresponding to cuts through the density plane in figure 11(a) along the $x$ - and the $y$-axis, respectively, which is compared to a Gaussian of the same height as the density peaks. It is found that the density profile has an isotropic Gaussian form for small distances from the origin $r \lesssim 0.1 / \rho^{1 / 2}$. For larger distances, however, i.e., where the density is of the order $\rho(\boldsymbol{r}) \lesssim \rho$, the density profile significantly deviates from a Gaussian form. In particular, we observe the establishment of "bridges" of higher density between neighbouring lattice sites, whereas the density is significantly lower between next-nearest neighbours. This 


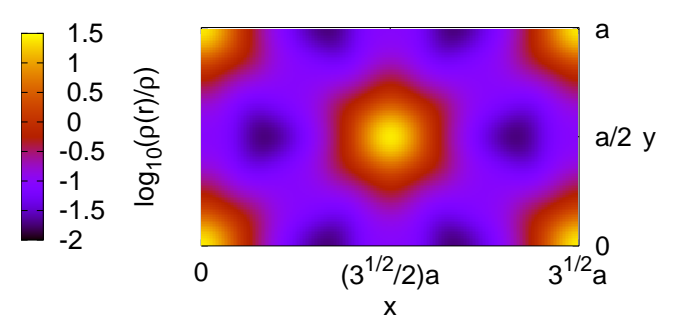

(a)

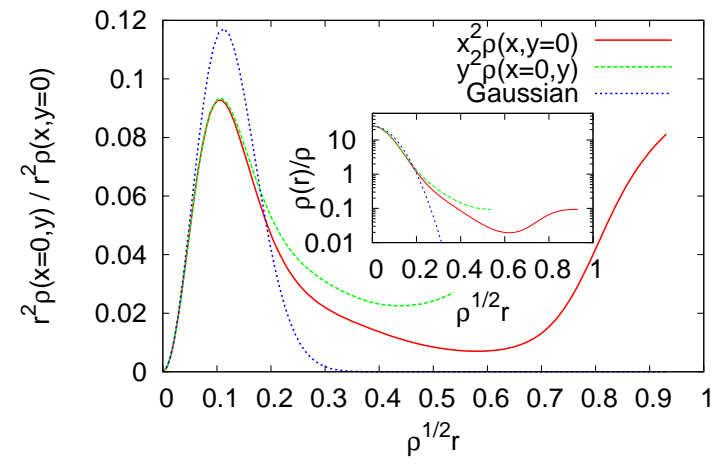

(b)

Figure 11. (a) The density profile $\rho(\boldsymbol{r})$ obtained from dynamical DFT using the SOT with the RY closure for $\Gamma=36$ which is close to freezing. (b) The quantity $r^{2} \rho(\boldsymbol{r} ; t \rightarrow \infty)$ along the straight line connecting two nearest neighbours $\left[y^{2} \rho(x=0, y ; t \rightarrow \infty)\right.$, i.e., in the [10]-direction] and along the line connecting two next-nearest neighbours $\left[x^{2} \rho(x, y=0 ; t \rightarrow \infty)\right.$, i.e., in the [11]-direction], both drawn from the center to the respective edge of the box in (a). The two curves are compared to a Gaussian of the same amplitude at $\boldsymbol{r}=0$. The inset displays the bare density along the same lines and the bare Gaussian.

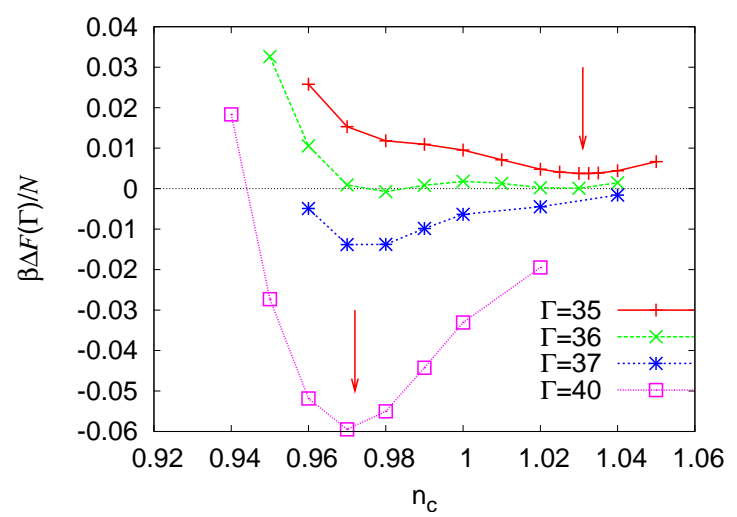

Figure 12. The difference in Helmholtz free energy per particle $\Delta F\left(n_{c} ; \Gamma\right) / N$ as a function of $n_{c}$ for different coupling constants $\Gamma=35,37,40$. The arrows indicate the positions of the minima.

counter-intuitive behavior was also found applying the MWDA to hard sphere crystals in three spatial dimensions [18]. However, computer simulations revealed that the behavior should be the opposite. Although we did not measure the density profiles of the twodimensional dipolar system in computer simulations, we expect a similar behavior: The probability density should be enhanced along the [11]-direction as compared to the [10]-direction.

We also performed the minimization procedure for different vacancy concentrations. In figure 12 we show the free energy difference $\Delta F\left(n_{c} ; \Gamma\right)$ as a function of $n_{c}$ for four different values of $\Gamma$. We find, that for crystals in equilibrium, i.e., for $\Gamma \gtrsim 36$, the 
equilibrium vacancy concentration is $1-n_{c} \approx 0.03$. However, the overheated crystal which is metastable for $31 \lesssim \Gamma \lesssim 36$ prefers a vacancy concentration of $1-n_{c} \approx-0.03$, implying interstitials instead of vacancies. We note that most of the point defects in the experimental realization of the dipolar system appear in pairs or in pairs of pairs as dislocations or pairs of dislocations, respectively [26].

\section{Discussion and concluding remarks}

In conclusion, we have demonstrated that density functional theory is able to quantitatively predict the freezing transition of a two-dimensional colloidal system with long-range $1 / r^{3}$-interactions in good agreement with experimental and simulation data. In complete analogy to systems in 3D, the appearance of long-range interactions requires the explicit inclusion of three-particle correlation functions of the liquid in the construction of the weighted density [13, 22. Furthermore, the predicted transition temperatures are very sensitive towards slight changes of the two- and three-particle correlation functions of the underlying fluid. A highly accurate input of the same is therefore crucial.

The obtained density functional can be used in future studies in order to approach more complicated situations such as crystals in confinement [18], under gravity [47, and crystal-fluid interfaces [18, 48]. By extending the static functional to Brownian dynamics [42, 43, 44, 45, 46], one may even address nonequilibrium situations. One possible problem to tackle is heterogeneous nucleation upon temperature quenches and subsequent crystal growth as outlined recently in Ref. [46].

\section{Acknowledgments}

We thank Norman Hoffmann for helpful discussions. This work has been supported by the DFG within the Collaborative Research Centre SFB TR6, "Physics of Colloidal Dispersions in External Fields", project section C3.

\section{References}

[1] Evans R. Adv. Phys., 28:143, 1979.

[2] Oxtoby D W. In J P Hansen, Levesque D, and Zinn-Justin J, editors, Liquids, Freezing and the Glass Transition, volume Session LI (1989) of Les Houches Summer Schools of Theoretical Physics, page 147, Amsterdam, 1991. North Holland.

[3] Singh Y. Phys. Rep., 207:351, 1991.

[4] Löwen H. Phys. Rep., 237:249, 1994.

[5] Löwen H. J. Phys.: Condens. Matter, 14:11897, 2002.

[6] Likos C N. Phys. Rep., 348:267-349, 2001.

[7] Rosenfeld Y. Phys. Rev. Lett., 63:980, 1989.

[8] Rosenfeld Y, Schmidt M, Löwen H, and Tarazona P. Phys. Rev. E, 55:4245, 1997.

[9] Schmidt M. J. Phys.: Condens. Matter, 15:S101, 2003.

[10] Curtin W A. J. Chem. Phys., 88:7050, 1988.

[11] Curtin W A and Ashcroft N W. Phys. Rev. A, 32:2909, 1985. 
[12] de Kuijper A, Vos W L, Barrat J-L, Hansen J-P, and Schouten J A. J. Chem. Phys., 93:5187, 1990.

[13] Likos C N and Ashcroft N W. Phys. Rev. Lett., 69:316, 1992.

[14] Zeng X C and Oxtoby D W. J. Chem. Phys., 93:2692, 1990.

[15] Rosenfeld Y. Phys. Rev. A, 42:5978, 1990.

[16] Tejero C F and Cuesta J A. Phys. Rev. E, 47:490, 1993.

[17] van Teeffelen S, Likos C N, Hoffmann N, and Löwen H. Europhys. Lett., 75:583, 2006.

[18] Ohnesorge R, Löwen H, and Wagner H. Europhys. Lett., 22:245, 1993.

[19] Ramakrishnan T V and Yussouff M. Phys. Rev. B, 19:2775, 1979.

[20] Laird B B and Kroll D M. Phys. Rev. A, 42:4810, 1990.

[21] Denton A R and Ashcroft N W. Phys. Rev. A, 39:4701, 1989.

[22] Likos C N and Ashcroft N W. J. Chem. Phys., 99:9090, 1993.

[23] Barrat J-L, Hansen J-P, and Pastore G. Mol. Phys., 63:747, 1988.

[24] Denton A R and Ashcroft N W. Phys. Rev. A, 39:426, 1989.

[25] Barrat J-L, Xu H, Hansen J-P, and Baus M. J. Phys. C., 21:3165, 1988.

[26] Zahn K, Lenke R, and Maret G. Phys. Rev. Lett., 82:2721, 1999.

[27] Lin S Z, Zheng B, and Trimper S. Phys. Rev. E, 73:066106, 2006.

[28] Haghgooie R and Doyle P S. Phys. Rev. E, 72:011405, 2005.

[29] Löwen H. Phys. Rev. E, 53:R29, 1996.

[30] Froltsov V A, Blaak R, Likos C N, and Löwen H. Phys. Rev. E, 68:061406, 2003.

[31] Zahn K, Méndez-Alcaraz J M, and Maret G. Phys. Rev. Lett., 79:175, 1997.

[32] Weeks J D. Phys. Rev. B, 24:1530, 1981.

[33] Hoffmann N, Likos C N, and Löwen H. J. Phys.: Condens. Matter, 18:10193, 2006.

[34] Hansen J-P and McDonald I R. Theory of simple liquids. Academic Press, London, 2nd edition, 1986.

[35] Rogers F J and Young D A. Phys. Rev. A, 30:999, 1984.

[36] Caillol J M, Levesque D, and Weis J-J. Mol. Phys., 44:733, 1981.

[37] Allen M P and Tildesley D J. Computer Simulation of Liquids. Clarendon Press, Oxford, 1987.

[38] Verlet L. Phys. Rev., 165:201, 1968.

[39] Baus M and Hansen J-P. Phys. Rep., 59:1, 1980.

[40] Rosenfeld Y, Levesque D, and Weis J-J. J. Chem. Phys., 92:6818, 1990.

[41] Froltsov V A, Likos C N, Löwen H, Eisenmann C, Gasser U, Keim P, and Maret G. Phys. Rev. E, 71:031404, 2005.

[42] Löwen H, Likos C N, Assoud L, Blaak R, and van Teeffelen S. Philos. Mag. Lett., 87:847, 2007.

[43] Marconi U M B and Tarazona P. J. Chem. Phys., 110:8032, 1999.

[44] Dzubiella J and Likos C N. J. Phys.: Condens. Matter, 15:L147, 2003.

[45] Archer A J and Evans R. J. Chem. Phys., 121:4246, 2004.

[46] van Teeffelen S, Likos C N, and Löwen H. Phys. Rev. Lett., 100:108302, 2008.

[47] Biben T, Ohnesorge R, and Löwen H. Europhys. Lett., 28:665, 1994.

[48] Marr D W and Gast A P. Phys. Rev. E, 47:1212, 1993. 